\title{
WEIGHTS, SHARP MAXIMAL FUNCTIONS AND HARDY SPACES ${ }^{1}$
}

\author{
BY JAN-OLOV STRÖMBERG AND ALBERTO TORCHINSKY
}

A considerable development of harmonic analysis in the last few years has been centered around a function space shown in a new light, the functions of bounded mean oscillation, and the weighted inequalities for classical operators. The new techniques introduced by C. Fefferman and E. M. Stein and B. Muckenhoupt are basic in these areas. It is our purpose here to develop some of these results in a very general setting, namely that of a metric space $(X, d)$ endowed with a doubling measure $d \mu$ and a weighted measure $d \nu=w d \mu$ with positive weight $w$. When there are a constant $c$ and a number $q>0$ such that if $B(x, r)=$ $\{y \in X: d(x, y) \leqslant r\}$ then $\mu(B(x, r t)) \leqslant c t^{q} \mu(B(x, r))$ for all $t \geqslant 1, r>0$ and $x \in X$ we say that $\mu$ satisfies the $D_{q}$ condition and that $\mu$ is doubling, or $\mu \in D_{\infty}$, when $\mu \in D_{q}$ for some $q$. We further assume that $\mu(B(x, r))$ is a continuous function of $r$ and that compactly supported continuous functions are dense in $L^{1}(d \mu)$. Because of the numerous applications of these results we feel that a detailed study is justified and a description of the new methodology needed to develop it follows.

For each $B(x, r)=B$ we define the median value $w_{B}$ as $\sqrt{t_{1} t_{2}}$ where $t_{1}=$ $\sup \{t>0: \mu\{x \in B: w(x)<t\} \leqslant \mu(B) / 2\}$ and $t_{2}=\inf \{t>0: \mu\{x \in B: w(x)$ $>t\} \leqslant \mu(B) / 2\}$. Then $w$ satisfies the $A_{\infty}$ condition, or $w \in A_{\infty}$, if $\nu(B) / \mu(B) \leqslant$ $c w_{B}$. When $w \in A_{\infty}, w^{-1 /(p-1)} \in A_{\infty}$ also for some $p>1$ and there is equivalent to saying that $w$ satisfies the usual $A_{p}$ condition, or $w \in A_{p}$. Aside from the trivial implications the conditions $A_{p}$ and $D_{q}$ are independent. For $A_{\infty}$ weights the following properties are obtained:

(1) $\left(\int_{B} w^{r} d \mu\right) / \mu(B) \sim\left(w_{B}\right)^{r}$ for $r=\beta_{1} \geqslant 1$ and $r=-\beta_{2}<0$;

(2) if $B_{1} \subseteq B$, then for some $\gamma_{i} \geqslant \beta_{i}$ and a constant $c$,

$$
c^{-1}\left(\mu\left(B_{1}\right) / \mu(B)\right)^{1+1 / \gamma_{2}} \leqslant \nu\left(B_{1}\right) / \nu(B) \leqslant c\left(\mu\left(B_{1}\right) / \mu(B)\right)^{1-1 / \gamma_{1}} ;
$$

(3) a strong version of the $\mathbf{P}$. Jones factorization holds, to wit, if $w$ satisfies (1) and (2) then $w=w_{1} w_{2}$ where both $w_{1}$ and $w_{2}$ also satisfy (1) and (2) with indices $\gamma_{1}-\epsilon$ and $\gamma_{2}+\epsilon$. In addition $w_{1}(x) \geqslant c w_{B}$ and $w_{2}(x) \leqslant c w_{B}$ for all $x$ in $B$.

The proof of (3) is too intricate to be described here but it requires the

Received by the editors May 5, 1980.

1980 Mathematics Subject Classification. Primary 43A15.

1 Research partly supported by NSF Grants. 
existence of Lip $\alpha$ functions in $X$ and some ideas, such as telescoping sequence of balls, which will also be used in considering the sharp maximal functions which are of interest to us. If $f$ is a locally summable function we set

$$
M^{\#} f(x)=\sup _{B} \inf _{c}\left(\int_{B}|f-c| d \mu\right) / \mu(B),
$$

and if $f$ is merely measurable we set

$$
M_{0, s}^{\#} f(x)=\sup _{B} \inf _{c} \inf \{A>0: \mu\{y \in B:|f(y)-c|>A\} \leqslant s \mu(B)\},
$$

where $c$ is a complex number, $x \in B$ and $0<s<1$.

THEOREM 1. If $s$ is sufficiently small and $\mu$ is doubling, then there are constants $c_{1}$ and $c_{2}$ such that $\mu\left\{y \in B:\left|f(y)-c_{B, f}\right|>\lambda, M_{0, s}^{\#} f(y) \leqslant \alpha\right\} \leqslant$ $c_{1} \exp \left(-c_{2} \lambda / \alpha\right) \mu(B)$.

THEOREM 2. If $s$ is sufficiently small and $\mu$ is doubling, then

$$
\left\|f-c_{f}\right\|_{L^{p}(d \mu)} \leqslant c\left\|M_{0, S}^{\#} f\right\|_{L^{p(d \mu)}}, \quad 0<p<\infty .
$$

In the present context a tempered distribution $f$ in $R^{n}$ is in the weighted Hardy space $H_{w}^{p}\left(R^{n}\right), 0<p<\infty$, if $M\left(F_{\phi}, x\right)=\sup _{|x-y| \leqslant t}\left|F_{\phi}(y, t)\right|$ is in the class $L_{w}^{p}\left(R^{n}\right)$ where $F_{\varphi}(y, t)=\left(f_{*} \phi_{t}\right)(y)$ is the extension of $f$ to $R_{+}^{n+1}$ by convolution with the dilations $\phi_{t}(y)=t^{-n} \phi(y / t)$ of a smooth function $\phi$ with nonvanishing integral. Denote $\|f\|_{H_{w}^{p}}=\left\|M\left(F_{\phi}\right)\right\|_{L_{w}^{p}}$. If $w$ is doubling (for notations and terminology see the previous note) then also the grand maximal function $f^{* *}(x)=\left\{\sup _{\phi} M\left(F_{\phi}, x\right): \phi\right.$ is smooth and $\left|x^{\sigma}(\partial / \partial x)^{\beta} \phi(x)\right| \leqslant 1, \sigma, \beta$ sufficiently large \} is also in $L_{w}^{p}\left(R^{n}\right)$, and consequently the $H_{w}^{p}\left(R^{n}\right)$ are intrinsically defined so that we may set $F_{\phi}(y, t)=F(y, t)$. Also when $w \in A_{\infty}$ the Lusin and Littlewood-Paley functions give equivalent norms in $H_{w}^{p}\left(R^{n}\right)$. Distributions in the Hardy spaces admit an atomic decomposition which we now describe. Given $1 \leqslant$ $r \leqslant \infty$ and an integer $N$ we say that $a$ is an $(r, N)$ atom if (1) supp $a \subseteq$ ball $B$, (2) $\|a\|_{L^{r}} \leqslant\left\|\chi_{B}\right\|_{L^{r}}$ and (3) $\int a(y) y^{\sigma} d y=0,|\sigma| \leqslant N$. Then the following is obtained.

THEOREM 3. Assume that $w \in D_{q}$, that $0<p \leqslant 1$ and that $\widetilde{N}=$ $[q n / p-1]$ where [] stands for the greatest integer function $\leqslant$. Then given $f$ in $H_{w}^{p}$ there is a sequence $\left\{a_{k}\right\}$ of $(\infty, N)$ atoms, $N \geqslant \widetilde{N}$, supported in $B_{k}$ such that $f=\Sigma \lambda_{k} a_{k}$ and $\|f\|_{H_{w}^{p}}^{p} \sim \Sigma\left|\lambda_{k}\right|^{p} w\left(B_{k}\right)$.

The convergence of the sum of $f$ is understood to take place in the $H_{w}^{p}$ metric and in the sense of distribution. The converse also holds.

THEOREM 4. Assume that $w$ is doubling, that $1<p<\infty$ and that $1<r$ $\leqslant \infty$. Let $f$ be in $H_{w}^{p}$. Then $f$ can be decomposed into a sum of $(r, N)$ atoms 
$f=\Sigma \lambda_{k} a_{k}$ with supp $a_{k} \subseteq B_{k}$ and such that $\left\|\Sigma \lambda_{k} \chi_{B_{k}}\right\|_{L_{w}^{p}} \leqslant c\|f\|_{H_{w}^{p}}$. The sum converges in the sense of distributions.

On the other hand let $1 \leqslant p<r \leqslant \infty$ and $\tilde{N}=[q n-1]$. When $r<\infty$ in addition suppose that $(*)\left(\int_{B} w^{(r / p)^{\prime}}\right) /|B| \leqslant c\left(\int_{B} w /|B|\right)^{(r / p)^{\prime}}$. Then for any sum $\Sigma \lambda_{k} a_{k}$ of $(r, N)$ atoms, $N>\widetilde{N}$, with supp $a_{k} \subseteq B_{k}$, we have that $\left\|\Sigma \lambda_{k} a_{k}\right\|_{H_{w}^{p}} \leqslant\left\|\Sigma \lambda_{A} \chi_{B_{k}}\right\|_{L_{w}^{p}}$. The following estimate for sums of characteristic functions of balls plays a crucial role in the proof of Theorem 2: $\left\|\Sigma \lambda_{k} \chi_{t B_{k}}\right\|_{L_{w}}^{p}$ $\left\langle c t^{\delta}\left\|\Sigma \lambda_{k} \chi_{B_{k}}\right\|_{L} \underset{w}{p}, \lambda_{k}>0, t>1\right.$, where the constant $\delta=\delta(w, p)$ is related to $p$ and the $A_{p}$ and $D_{q}$ conditions on $w$.

THEOREM 5. Let $1 \leqslant p<r \leqslant \infty$; assume that $w \in A_{\infty} \cap D_{q}$ and that (*) holds. Further assume that $N>[q n-1]$ and that $T$ is a linear operator which maps each $(r, N)$ atom $a_{k}$ into a sum of $(r, N)$ atoms $\Sigma 2^{-j \gamma} a_{k, j}$ with supp $a_{k, j}$ $\subseteq 2^{j} B_{k}$. Then $T$ is a bounded operator on $H_{w}^{p}$ provided $\gamma>\delta=\delta(w, p)$. Similarly if $T$ is subadditive and the $a_{k, j}$ are $(r, 0)$ atoms, then $T$ maps $H_{w}^{p}$ boundedly into $L_{w}^{p}$. Clearly the former remark applies to multiplier and singular integral transforms and the latter to pseudo-differential operators. In this general context we would like to point out that complex interpolation can be carried out quite explicitly with the use of atoms and that finite linear combinations of atoms are dense in the Hardy spaces.

Also central to the theory of weighted Hardy spaces is the extension of an inequality due to R. Fefferman, Stein and Strömberg in the unweighted case.

THEOREM 6. Let $f$ be a finite linear combination of atoms, $F(y, t)$ as above, and $g \in L_{\text {Loc }}^{1}\left(R^{n}\right)$. Then for $A \geqslant 1$ we have

$$
\left|\int f g\right| \leqslant c\left(\int M(F, x)^{1 / A}\left(M_{w^{A}}^{\#} g\right)(x)^{1 / A} w(x) d x\right)^{A}
$$

where $w \in D_{q}$ and

$$
\left(M_{w^{A}}^{\#} g\right)(x)=\sup _{x \in B}\left(\inf _{P}\left[\frac{1}{w(B)^{A}} \int_{B}|g-P|\right]\right)
$$

$P=$ polynomial of degree (nqA - 1). This inequality allows us to show, for instance, that $\left(H_{w}^{p}\right)^{*}=\left\{g \in L_{L o c}^{\prime}\left(R^{n}\right):\left(M_{w_{1}^{1 / p}}^{\#} g\right) \in L^{\infty}\right\}$ when $0<p \leqslant 1$ and $\left\{g \in L_{\text {loc }}^{1}\left(R^{n}\right):\left\|M_{w}^{\#} g\right\|_{L} p_{w}^{\prime}<\infty, 1 / p+1 / p^{\prime}=1\right\}$ when $p>1$.

\section{REFERENCES}

A. P. Calderón (1976), Inequalities for the maximal function relative to a metric, Studia Math. 57, 297-306. 
A. P. Calderón and A. Torchinsky (1975), Parabolic maximal functions associated with a distribution, Adv. in Math. 16, 1-64; (1977), part II, Adv. in Math. 24, 101-171. 269-274.

R. R. Coifman (1974), A real variable characterization of $H^{p}$, Studia Math. 51,

R. R. Coifman and C. Fefferman (1974), Weighted norm inequalities for maximal functions and singular integrals, Studia Math. 51, 241-250.

R. R. Coifman and G. Weiss (1977), Extensions of Hardy spaces and their use in analysis, Bull. Amer. Math. Soc. 83, 569-645. 137-193.

C. Fefferman and E. M. Stein (1972), $H^{p}$ spaces of several variables, Acta Math. 129,

J. Garcia-Cuerva (1979), Weighted $H^{p}$ spaces, Dissertationes Math. 162, 63 pages.

P. Jones, Factorization of $A_{p}$ weights (preprint).

R. Latter and A. Uchiyama (1979), The atomic decomposition for parabolic $H^{p}$ spaces, Trans. Amer. Math. Soc. 253, 391-398.

B. Muckenhoupt (1972), Weighted norm inequalities for the Hardy maximal function, Trans. Amer. Math. Soc. 167, 207-226.

J.-O. Strömberg (1979), Bounded mean oscillation with Orlicz norms and duality of Hardy spaces, Indiana Math. J. 28, 511-544.

(1979), Non-equivalence between two kinds of conditions on weight functions, Proc. Sympos. Pure Math., vol. 35, Part I, Amer. Math. Soc., Providence, R.I., pp. 141-148. (preprint).

J.-O. Strömberg and A. Torchinsky (1980), Weights and sharp maximal functions

DEPARTMENT OF MATHEMATICS, PRINCETON UNIVERSITY, PRINCETON, NEW JERSEY 08544

DEPARTMENT OF MATHEMATICS, INDIANA UNIVERSITY, BLOOMINGTON, INDIANA 47405 